OPEN ACCESS

Edited by:

Won-Seok Kim,

Seoul National University Bundang

Hospital, South Korea

Reviewed by:

Kathryn S. Hayward,

The University of Melbourne, Australia

SuYeon Kwon,

Ewha Womans University Seoul

Hospital, South Korea

${ }^{*}$ Correspondence:

Shashwati Geed

shashwati.geed@medstar.net

Specialty section:

This article was submitted to

Stroke,

a section of the journa

Frontiers in Neurology

Received: 01 March 2021

Accepted: 06 May 2021

Published: 08 June 2021

Citation:

Geed S, Feit P, Edwards DF and Dromerick AW (2021) Why Are Stroke Rehabilitation Trial Recruitment Rates in Single Digits?

Front. Neurol. 12:674237. doi: 10.3389/fneur.2021.674237

\section{Why Are Stroke Rehabilitation Trial Recruitment Rates in Single Digits?}

\author{
Shashwati Geed ${ }^{1,2 *}$, Preethy Feit ${ }^{2}$, Dorothy F. Edwards ${ }^{3}$ and Alexander W. Dromerick ${ }^{1,2,4}$ \\ ${ }^{1}$ Center for Brain Plasticity and Recovery, Department of Rehabilitation Medicine, Georgetown University Medical Center, \\ Washington, DC, United States, ${ }^{2}$ MedStar National Rehabilitation Hospital, Washington, DC, United States, ${ }^{3}$ Department of \\ Kinesiology and Occupational Therapy, University of Wisconsin, Madison, WI, United States, ${ }^{4}$ Department of Neurology, \\ Georgetown University Medical Center, Washington, DC, United States
}

Background: Recruitment of patients in early subacute rehabilitation trials ( $<30$ days post-stroke) presents unique challenges compared to conventional stroke trials recruiting individuals $>6$ months post-stroke. Preclinical studies suggest treatments be initiated sooner after stroke, thus requiring stroke rehabilitation trials be conducted within days post-stroke. How do specific inclusion and exclusion criteria affect trial recruitment rates for early stroke rehabilitation trials?

Objectives: Provide estimates of trial recruitment based on screening and enrollment data from a phase II early stroke rehabilitation trial.

Methods: CPASS, a phase II intervention trial screened ischemic stroke patients in acute care (18-months, $N=395)$ and inpatient rehabilitation (22-months, $N=673$ ). Patients were stratified by upper extremity (UE) impairment into mild (NIHSS motor arm =0, 1); moderate $(\mathrm{NIHSS}=2,3)$; severe $(\mathrm{NIHSS}=4$ ) and numbers of patients disqualified due to CPASS exclusion criteria determined. We also examined if a motor-specific evaluation (Action Research Arm Test, ARAT) increases the pool of eligible patients disqualified by the NIHSS motor arm item.

Results: CPASS recruitment in acute care (5.3\%) and inpatient rehabilitation (5\%) was comparable to prior trials. In acute care, a short stay (7-17-days), prior stroke (13.5\% in moderately; $13.2 \%$ in severely impaired) disqualified the majority. In inpatient rehabilitation, the majority (40.8\%) were excluded for "too mild" impairment. The next majority were disqualified for reaching inpatient rehabilitation "too late" to participate in an early stroke trial (15\% in moderately; $24 \%$ in severely impaired). Mean ARAT in the "too mild" showed significant impairment and potential to benefit from participation in select UE rehabilitation trials.

Conclusions: Screening of ischemic stroke patients while they are still in acute care is crucial to successful recruitment for early stroke rehabilitation trials. A significant proportion of eligible patients are lost to "short length of stay" in acute care, and arrive to inpatient rehabilitation "too late" for an early rehabilitation trial. Additional screening of mildly impaired patients using a motor function specific scale will benefit the trial recruitment and generalizability.

Trial Registration Number: http://www.clinicaltrials.gov Identifier: NCT02235974.

Keywords: prospective study, rehabilitation, clinical trial, stroke, trial design 


\section{INTRODUCTION}

Stroke rehabilitation trials have conventionally focused on individuals whose motor recovery has plateaued at more than 3-6 months post-stroke (1-5) so that spontaneous post-stroke recovery does not confound intervention-related recovery (6). However, preclinical studies increasingly suggest that post-stroke rehabilitation intervention trials need to be conducted within days of stroke onset for better neuromotor outcomes (2, 79). In traditional stroke rehabilitation trials, investigators have a long window of recruitment often lasting up to several years post stroke because patients who are more than 6-months post stroke are included. Additionally, rehabilitation trialists have conventionally recruited from outpatient stroke clinics and community centers that offer multiple opportunities with repeated contact to enroll the same individuals.

To enable acute ( $<7$ days) and early-subacute (8-90 days post stroke) trials $(10,11)$, rehabilitation trial recruitment methods need to adapt by shifting into the acute care and inpatient rehabilitation settings where patient stays are limited for 2 weeks post stroke on average (12). Thus, early stroke rehabilitation trialists have a brief window of identifying and enrolling eligible patients in trials. This brief period coincides with a particularly confusing time in patients' lives given their recent stroke diagnosis and the long commitment required of them to participate in a stroke rehabilitation trial with a typical follow up at 12-months post randomization $(5,7,13-15)$. Even small efficiencies in patient screening and enrollment in this scenario can make large differences in the eventual trial recruitment rates and costs. However, there are no data at present that give reliable estimates of how trial inclusion and exclusion criteria affect the pool of eligible patients in a US healthcare setting for an early stroke rehabilitation trial.

Lasagna's law (16) notes that "the incidence of any disease decreases sharply as soon as a clinical trial begins and returns to its original level as soon as the trial is completed." No matter how conservative one is about their recruitment goals, it is difficult to recruit study participants according to expectations. Incorrect estimates of trial recruitment lead to delays in study completion, abandoned studies, and mismanagement of research funds (17). Underpowered studies increase the probability of type II errors affecting study integrity and validity. Inadequate trial inclusion/exclusion criteria negatively affect generalizability and internal validity of the study. Thus, reliable estimates of trial recruitment rates are central to any trial's planning and logistics.

Here, we report screening and enrollment data from the phase II Critical Periods After Stroke Study (CPASS), an early stroke rehabilitation trial designed to identify optimal timing of upper extremity (UE) motor rehabilitation after stroke $(7,18)$. CPASS recruited individuals within 30 days of stroke and followed participants up to 12 months post randomization. CPASS screening data are from an urban safety-net acute-care hospital and an inpatient rehabilitation setting that used StrokeNet (19) resources. We also report the trial inclusion/exclusion criteria that most affected trial recruitment rates to help future investigators re-evaluate their own trial inclusion and exclusion to aid trial recruitment. Finally, UE stroke rehabilitation trial screening often relies on a prescreen (an easily available measure of motor function from medical history or charts to filter the patients for evaluation with the full trial inclusion/exclusion criteria), e.g., scores on the National Institutes of Health (NIHSS) Stroke Scale items $(2,20)$. Using CPASS data, we asked if stroke rehabilitation trial recruitment rates would benefit from using a motor function-specific screening tool over the commonly used NIHSS. This is a first report to our knowledge that highlights the typical characteristics of patients recruited or excluded for an early-subacute stroke neurorehabilitation trial in the US healthcare setting.

\section{METHODS}

Study procedures were approved by the Institutional Review Board at the MedStar Health Research Institute. The trial was registered at http://www.clinicaltrials.gov (Identifier: NCT02235974). Registry data from inpatients at MedStar Washington Hospital Center and MedStar National Rehabilitation Hospital (NRH) were used. The registry identifies potential participants for post-acute stroke studies at $24-48 \mathrm{~h}$ after admission. Data from neuroimaging-confirmed ischemic stroke survivors were extracted; additionally, data on the earliest available National Institutes of Health Stroke Scale (NIHSS) score, demographics, thrombectomy status, tissue plasminogen activator (tPA) administration, duration of stay, and discharge location were extracted. Patients entering inpatient rehabilitation at $\mathrm{NRH}$ are evaluated using the Action Research Arm Test (ARAT), a UE motor function specific assessment (21-24) as part of their intake evaluation; these ARAT scores were also extracted. We report CPASS screening data for an 18-month (02/2016 - 07/2017) duration from acute care, and 22 -month duration $(06 / 2015$ - 04/2017) from the inpatient rehabilitation setting.

UE rehabilitation trials typically stratify enrolled participants using severity of UE motor impairment (2, 5, 7, 14, 25). Therefore, to examine the effect of motor severity on trial eligibility rates, we used NIHSS arm motor score to categorize $\mathrm{UE}$ impairment into mild (NIHSS $=0,1)$, moderate $(\mathrm{NIHSS}=2$, $3)$, or severe (NIHSS $=4$ ) impairment (12).

To examine the impact of other trial inclusion/exclusion criteria on trial qualification rate, we used the medical, disability, and social support criteria from CPASS (Table 1). CPASS criteria are similar to multiple major stroke rehabilitation trial inclusion/exclusion criteria, including, VECTORS (2), ICARE (5), EXCITE (14), and LEAPS (4), and TRANSPORT-2.

Even a single exclusion criterion will put the patient out of the "qualified" pool of participants during screening. We followed a similar strategy to quantify patients excluded from the pool of eligible patients, but it is likely a patient in a given category has multiple exclusionary characteristics. Further, not all patients qualifying for the trial will eventually be enrolled. We therefore report the actual enrollment numbers in CPASS from acute care and inpatient rehabilitation to give trialists an estimate of the total numbers of patients screened for the number enrolled in the trial. Specifically, the results report the numbers of patients excluded due to each trial exclusion criteria from the acute care and inpatient rehabilitation screening. To determine 
TABLE 1 | CPASS trial inclusion and exclusion criteria.

\section{Inclusion criteria}

1. Ischemic or hemorrhagic stroke (with confirmatory neuroimaging) within 28 days of admission to inpatient rehabilitation

2. Age $\geq 21$ years

3. Able to participate in first study-related treatment session within 30 days of stroke onset

4. Able to participate in all study-related activities, including 1 year follow up and blood draws

5. Persistent hemiparesis leading to impaired UE function as indicated by a score $\geq 1$ on the NIHSS motor arm score, and motor impairment judged clinically appropriate as defined by one or more of the following:

a. Proximal UE voluntary activity indicated by a score of $\geq 3$ on the upper arm item of the motor assessment scale; wrist and finger movements are not required

b. Manual muscle test (MMT) score $\geq 2$ on shoulder flexion and either elbow flexion or extension or

c. Active range of motion (AROM) to at least $50 \%$ of range in gravity eliminated position for shoulder flexion or abduction, and for any of the following motions: elbow flexion, elbow extension, wrist flexion, wrist extension, finger flexion or finger extension

6. Score of $\leq 8$ on the Short Blessed Memory Orientation and Concentration scale

7. Follows 2-step commands

8. No upper extremity injury or conditions that limited use prior to the stroke

9. Pre-stroke independence: Modified Rankin Scale score of 0 or 1

\section{Exclusion criteria}

1. Inability to give informed consent.

2. Prior stroke with persistent motor impairment or other disabling neurologic conditions such as multiple sclerosis, Parkinsonism, ALS, dementia requiring medication.

3. Rapidly evolving motor function.

4. Clinically significant fluctuations in mental status in the 72 hours prior to randomization.

5. Hemispatial neglect as determined by an asymmetry $>3$ errors on the Mesulam symbol cancellation test.

6. Not independent prior to stroke (determined by scores of $<95$ on Barthel Index or $>1$ on modified Rankin scale.

7. Dense sensory loss indicated by a score of 2 on NIHSS sensory item.

8. Ataxia out of proportion to weakness in the affected arm as defined by a score $\geq 1$ on the NIHSS limb ataxia item.

9. Active or prior (within 2 years) psychosis.

10. Active or prior (within 2 years) substance abuse.

11. Not expected to survive 1 year due to other illnesses (cardiac disease, malignancy, etc.).

21. Received UE botulinum toxin within 6 months (other meds do not exclude).

the added benefit of using a UE motor-function specific scale on trial qualification rate, we examined the ARAT, in patients that were disqualified from CPASS due to their UE being "too mild" (NIHSS motor arm score $<1$ ). Descriptive statistics on the ARAT scores in patients with NIHSS motor arm score $<1$ were computed.

\section{RESULTS}

\section{In Acute Care, Short Length of Stay and Prior Stroke Lead to the Most Exclusions}

We identified 395 ischemic stroke survivors with an available NIHSS motor arm score from the acute care registry over the 18month screening duration. Mean NIHSS total score was $9.1 \pm$
TABLE 2 | Length of Stay and Discharge locations in Acute Care and Inpatient Rehabilitation.

\begin{tabular}{|c|c|c|c|c|c|c|}
\hline & \multicolumn{2}{|c|}{ Mild } & \multicolumn{2}{|c|}{ Moderate } & \multicolumn{2}{|c|}{ Severe } \\
\hline & Acute & Inpatient & Acute & Inpatient & Acute & Inpatient \\
\hline $\begin{array}{l}\text { Length of stay } \\
\text { (days) }\end{array}$ & 6.9 & 13.7 & 13.6 & 20.5 & 17.2 & 32.1 \\
\hline $\begin{array}{l}\text { Discharge home, } \\
\text { no service (\%) }\end{array}$ & 36.7 & 45.9 & 10.7 & 32.5 & 6.2 & 16.3 \\
\hline $\begin{array}{l}\text { Discharge home, } \\
\text { home health (\%) }\end{array}$ & 11.3 & 5.8 & 4.0 & 2.5 & 6.2 & 4.9 \\
\hline $\begin{array}{l}\text { Discharge home, } \\
\text { outpatient OT/PT } \\
(\%)\end{array}$ & 5.1 & 30.2 & 1.3 & 37.5 & 0.0 & 28.5 \\
\hline $\begin{array}{l}\text { Acute inpatient } \\
\text { rehabilitation (\%) }\end{array}$ & 34.0 & 0.7 & 49.3 & 0.8 & 30.8 & 2.4 \\
\hline $\begin{array}{l}\text { Subacute } \\
\text { rehabilitation/SNF } \\
(\%)\end{array}$ & 9.4 & 13.3 & 17.3 & 19.2 & 32.3 & 35.0 \\
\hline $\begin{array}{l}\text { Another acute } \\
\text { care hospital (\%) }\end{array}$ & 0.0 & 3.6 & 2.7 & 6.7 & 4.6 & 12.2 \\
\hline Hospice (\%) & 0.8 & 0.2 & 4.0 & 0.8 & 0.0 & 0.0 \\
\hline Death (\%) & 1.6 & 0.2 & 9.3 & 0.0 & 20.0 & 0.8 \\
\hline Other (\%) & 0.0 & 0.0 & 1.3 & 0.0 & 0.0 & 0.0 \\
\hline
\end{tabular}

9.3. Of these, $46.6 \%$ were male, and $77.2 \%$ identified as African American $(12.3 \%$ as White, and $1.4 \%$ as Asian with the rest Unknown or Refused to identify). Of the 395 patients, $21.2 \%$ received tPA, and $8.2 \%$ received a thrombectomy. Examining the degree of motor impairment, $64.8 \%$ had mildly impaired UE (NIHSS motor arm $=0$ or 1 ), $18.3 \%$ had moderately impaired UE (NIHSS motor arm $=2$ or 3 ), and $15.6 \%$ had severe UE impairment (NIHSS motor arm =4). Overall, $5.3 \%$ of the 395 individuals identified in screening eventually enrolled in CPASS from acute care.

To determine the average window of time to enroll patients, we examined patients' average length of stay. The length of stay in acute care and discharge locations for the three groups (mild, moderate, and severely impaired) is shown in Table 2. Length of stay correlated with degree of UE impairment, mild impairment $=6.9 \pm 6.9$ days, moderate $=13.6 \pm 15.4$ days, and severe $=$ $17.2 \pm 13.1$ days. Only $34 \%$ of the mildly impaired, $49 \%$ of the moderately impaired, and $31 \%$ of the severely impaired patients were discharged to an inpatient rehabilitation facility, which opens a second window to recruit these patients if the trialist implements screening resources at acute care and inpatient rehabilitation. $83 / 395$ ischemic stroke patients screened in this acute care report were admitted to inpatient rehabilitation at $\mathrm{NRH}$ and are included in the data on inpatient rehabilitation screening data as well to maintain integrity of the independent screening numbers at acute care and inpatient rehabilitation. A majority (54\%) were discharged home or to a skilled nursing facility. Unless a trial budgets for outreach to enroll patients discharged home, this large majority of potentially eligible patients may be entirely lost to trial enrollment if not recruited during their short hospital stay (6-17 days). 


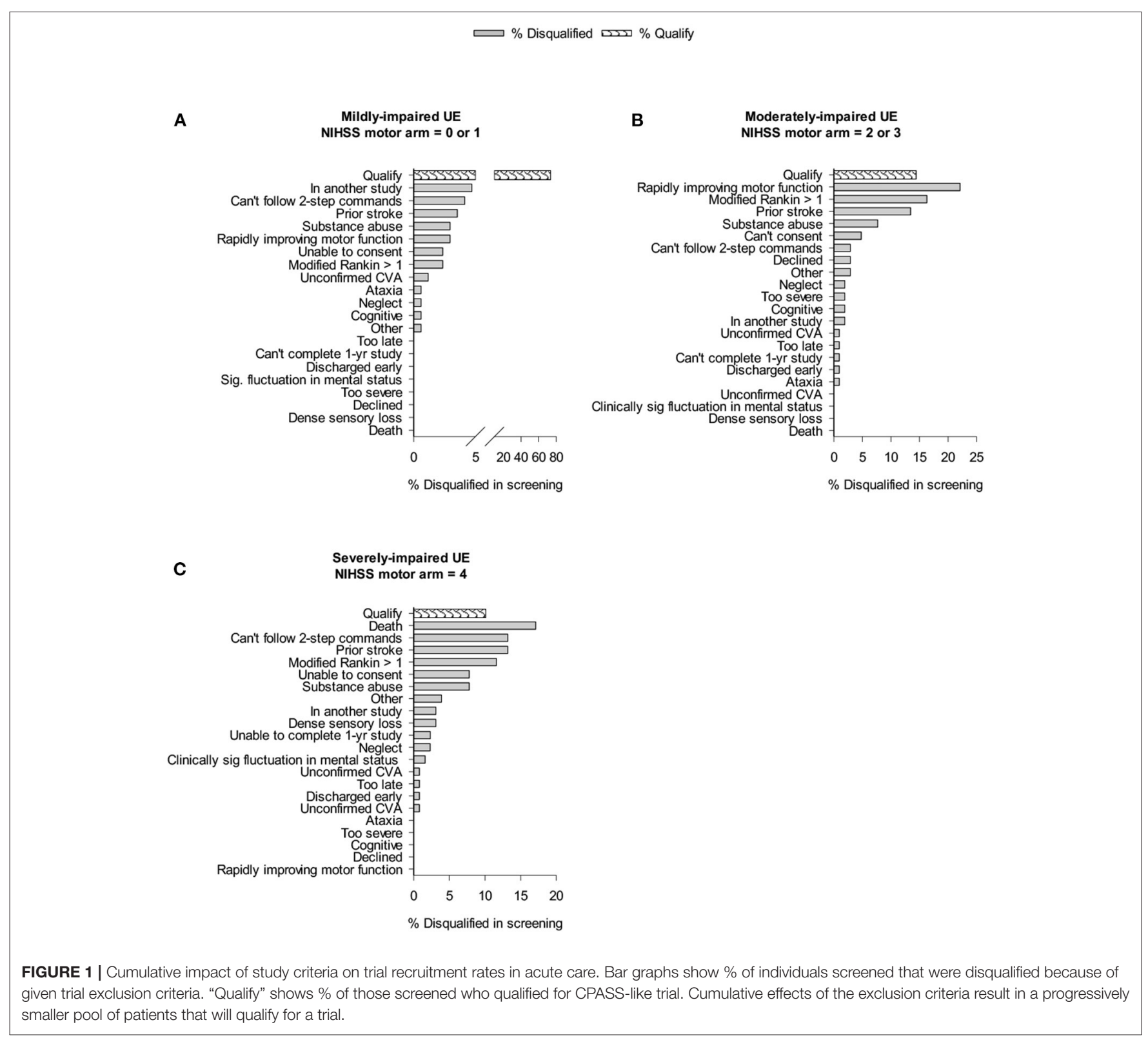

Figure 1 shows the impact of trial exclusion criteria on the pool of eligible patients from the mild, moderate, and severe UE impairment groups. In patients with mild impairment (Figure 1A), 73.5\% would potentially qualify for motor studies recruiting subjects with NIHSS motor arm score $=0$ or 1 . Examination of general medical, disability, and social criteria showed that main reasons for ineligibility include "in another study" (4.7\%), and "inability to follow 2 -step commands" (4.1\%). In patients with a moderately-impaired arm (Figure 1B), a mere $14.4 \%$ (3.7\% of all ischemic stroke patients) would qualify for a CPASS-like UE trial. In this moderately-impaired cohort, "rapidly improving motor function" (22\%), "disabled prior to stroke" (16.3\%), and "prior stroke with persistent impairments" (13.5\%) were the most frequent exclusions. In the severely impaired group (Figure 1C), 89.9\% of patients were eliminated by medical, disability, and social criteria. Patients with severe
UE impairment were most frequently excluded by prior stroke (13.2\%), or pre-stroke disability (11.6\%). Overall, $64 \%$ of patients with moderately-impaired (and $70 \%$ of those with severely impaired) UE's were excluded not by stroke, but rather inability to consent, substance abuse, prior stroke, pre-stroke disability, or rapid clinical improvement.

\section{At Inpatient Rehabilitation, Arriving Too Late, Mild Impairment, and Prior Stroke Lead to the Most Exclusions}

We identified 673 ischemic stroke survivors with an available NIHSS motor arm score during the 22-month screening for CPASS. Mean NIHSS total score was $6.6 \pm 5$. Of these $53.9 \%$ were male, $65.1 \%$ identified as African American, $16.8 \%$ as White, 0.9 and remaining as Asian, Other, or Refused to 


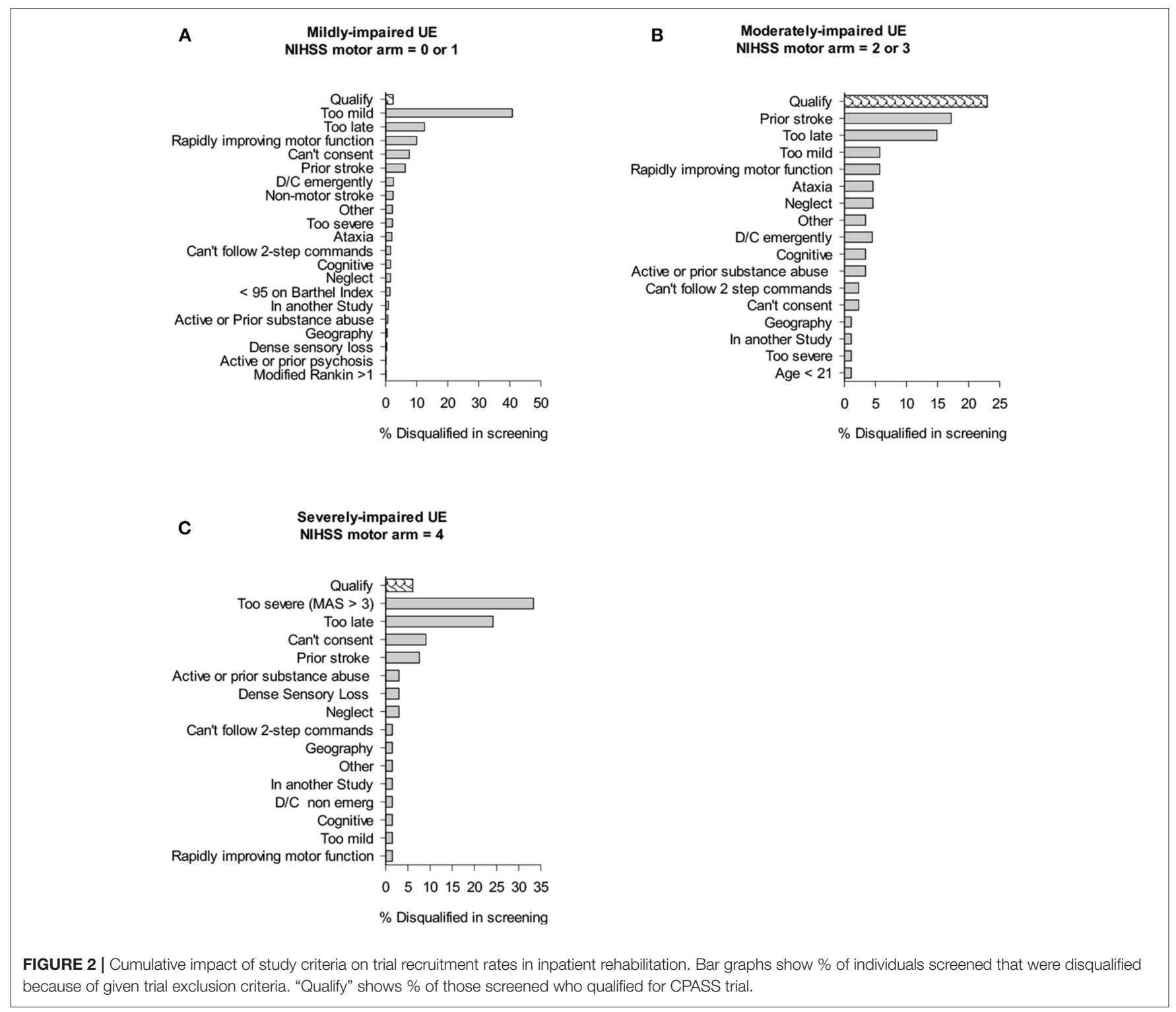

identify. Overall, $5 \%$ of the 673 individuals screened enrolled in CPASS.

Examining the degree of motor impairment, 62.1\% showed Mild, 19.7\% Moderate, and 18.2\% showed severe UE impairment. Average length of stay correlated with the degree of motor impairment; patients with mild impairment were inpatients for $13.7 \pm 13.4$ days, moderate impairment for $20.5 \pm 15.6$ days, and patients with severe impairment were inpatients for $32.1 \pm$ 20.4 days. Discharge locations by degree of motor impairment are shown in Table 2.

Figure 2 shows the impact of trial exclusion criteria on the available pool of qualified patients for the UE trial. Of all the mildly impaired participants, $40.8 \%$ of patients in inpatient rehabilitation were excluded because their impairment was too mild for the subacute intervention study (NIHSS motor arm score $=0$ or 1$)$. The next largest group (12.5\%) was disqualified for being "too late," i.e., they arrived at inpatient rehabilitation too late and would not receive the first study-related treatment session within 30-days of stroke onset. Ten percentage of mild inpatients were disqualified because their arm was recovering too rapidly for the context of this trial, e.g., the chart sheet recorded NIHSS motor arm item score $=2$ but in-person screening showed little to no deficit in the UE. The next largest group, $7.6 \%$ were disqualified for being unable to consent because of language issues, inability to understand the consent process, or aphasia. Lastly, "prior stroke" disqualified $6.3 \%$ of the patients. Of all the moderately impaired patients (NIHSS $=2$ or 3 ), the largest percentage (17.2\%) were disqualified because of a prior stroke. The next largest group (14.9\%) were disqualified because they arrived at inpatient rehabilitation "too late." Of all the severely impaired patients, the largest disqualification rate (33.3\%) came from patients being too severe for the CPASS trial (enrolling mild-moderately impaired), and $24.2 \%$ of patients were disqualified for being "too late" to inpatient rehabilitation. 


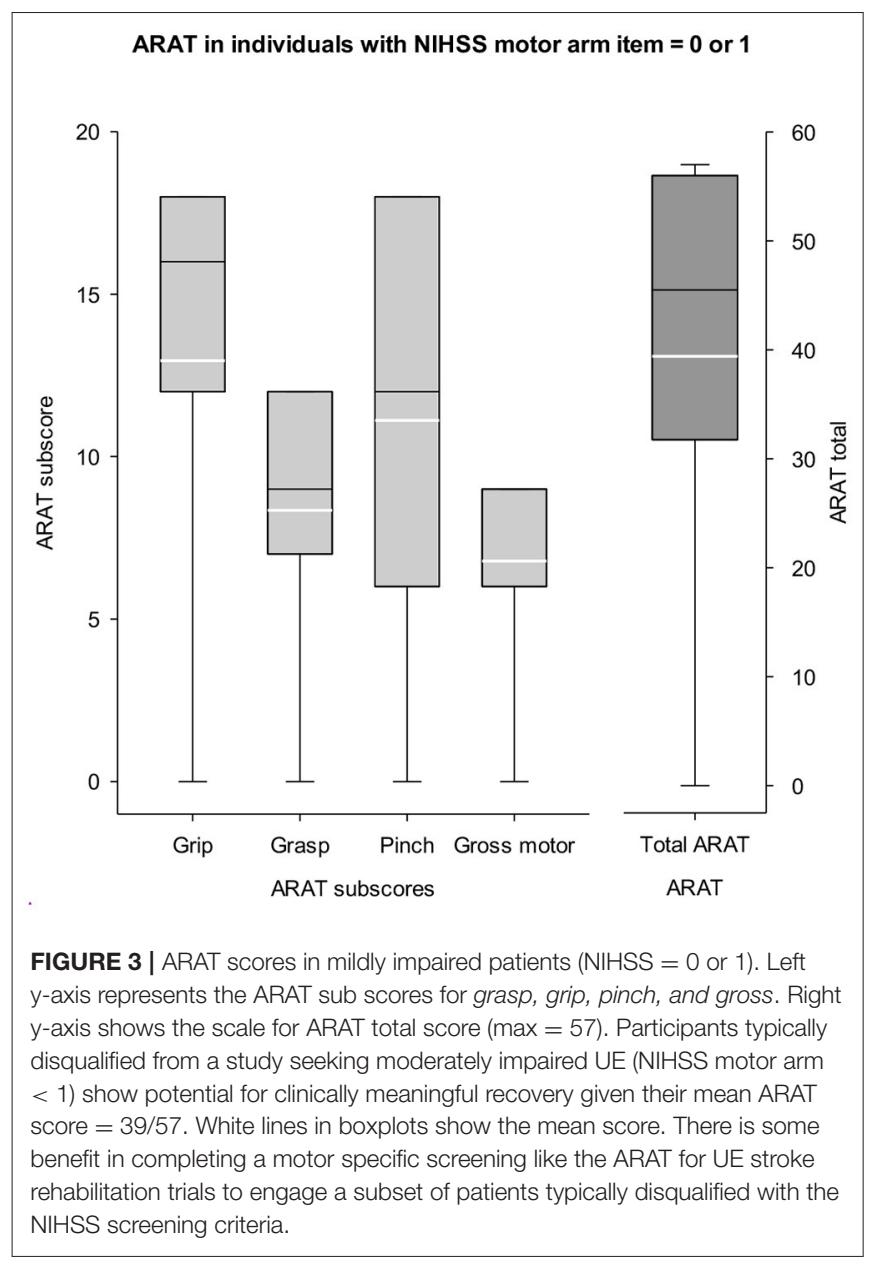

\section{Screening With ARAT Instead of NIHSS Motor Arm Item Benefits Trial Recruitment}

A large percentage of all patients admitted to acute care $(64.8 \%)$ and inpatient rehabilitation $(62.1 \%)$ that were screened for CPASS were disqualified for what would be classified as a "mildly impaired arm (NIHSS $=0$ or 1)." These patients would be automatically disqualified from UE stroke rehabilitation trials if screening were completed using only the NIHSS motor arm item and CPASS-like rehabilitation trial Inclusion/Exclusion criteria. Given the large percentages disqualified due to a "too mild" score on NIHSS motor arm item, we evaluated if there is any added benefit in numbers of patients qualifying for a UE stroke study if screening were done using a dedicated motor function specific scale like the ARAT. The distribution of ARAT scores in a random selection of 389/438 patients screened at inpatient rehabilitation is shown in Figure 3. Mean ARAT score for the affected UE was $39.41 \pm 18.6$, and $52.7 \pm 9.9$ for the unaffected UE $(\max$ score $=57)$.

\section{DISCUSSION}

Of the 395 ischemic stroke patients screened in acute care over 18 months, $5.3 \%$ were enrolled in CPASS. On the inpatient rehabilitation side, of the 673 ischemic stroke patients screened over 22 months, 5\% were enrolled in CPASS. These single-digit recruitment rates are comparable with major stroke rehabilitation trials. ICARE (5) recruiting patients in the subacute stage screened 11,051 patients to randomize 361 patients (recruitment rate of 3.2\%), EXCITE recruiting patients in the subacute phase $(14,26)$ screened 3,626 patients to randomize 222 patients (6.1\%), AVERT (17) screened 25,237 patients in the hyperacute stage, to randomize $2,104(8.3 \%)$. Thus, trial recruitment rates have remained in the single-digits for a majority of large multisite stroke rehabilitation trials.

Randomized controlled trials are the gold standard for evaluating treatment effectiveness. Major stroke rehabilitation trials so far have shown only marginal effectiveness in improvement of motor function compared to the standard of care $(2,5,27-36)$. In the US, a stroke occurs every $40 \mathrm{sec}(37)$; most individuals survive but with long-term motor impairments that seriously limit independent living. Nearly two thirds of stroke survivors are unable to use their affected arm even at 6 months post stroke (38-40). Thus, there is an urgent need for effective UE neurorehabilitation. Single-digit recruitment rates significantly slow down translation of preclinical to phase II/III studies while increasing the costs of stroke rehabilitation trials. Stroke trial recruitment rates have remained unchanged, in single digits between 1990 and 2014 (41) necessitating a systematic examination of how specific trial inclusion/exclusion criteria affect recruitment.

Recruitment logistics for acute-and early subacute stroke rehabilitation trials ( $<30$-days post) contrast sharply with conventional stroke rehabilitation trials typically conducted in the chronic phase (>6-months post-stroke). This early trial recruitment is challenging because patients are admitted for short duration (6-17 days). Patients' medical status changes rapidly during this time including their degree of motor arm impairment as shown by the large fractions of patients excluded because of a rapidly recovering UE. Correctly estimating if future motor impairment will be suitable for the trial, given patients' current motor function status is difficult $(42,43)$. This is especially relevant in the neurorehabilitation where interventions involve daily sessions for weeks and follow-ups at 12-months post-stroke $(5,7,13-15,26,44)$.

Given the unique challenges in recruiting for neurorehab studies early after stroke, we presented prospectively collected estimates of how specific trial inclusion and exclusion criteria affect trial recruitment rates. Based on our data, we also provide recommendations for best practices in optimizing trial inclusion/exclusion criteria to strike a balance between trial generalizability and maintaining a homogenous enough sample in the trial (Box 1). Importantly, multisite trials need to conduct thorough screening of potential recruitment sites prior to commencing trials such as CPASS to ensure that the site has adequate patient throughput and to identify potential site-specific barriers to recruitment (2). Patients' length of stay, transfer from acute care to inpatient rehabilitation or community rehabilitation services affects access to patients for recruitment. 
BOX 1 | Recommendations for best practices in screening for acute and early subacute trials.

1. Prescreening with a motor function specific tool like SAFE or ARAT may be better than NIHSS motor arm item alone, especially for the mildly impaired patients as it leads to a large percentage of exclusions.

2. Patients excluded for being too mild based on NIHSS criteria alone should be considered for inclusion in trials as their mean ARAT score $(39.4 \pm 18.6)$ is substantially lower than the ARAT ceiling of 57 . The difference between mean ARAT in mildly-impaired and ARAT's ceiling is greater than the ARAT $M C I D$, suggesting these patients are likely to benefit from participating in the trial, and show clinically meaningful improvement.

3. Screening and recruitment efforts must begin in acute care as a large percentage arrive to inpatient rehabilitation too late to be enrolled in early stroke trials.

4. Prior strokes lead to a large percentage of exclusions. Trialists need a pragmatic definition for which patients should be excluded given the large percentages of second strokes. For phase II/III motor function intervention trials, "prior stroke without residual motor impairment" may be acceptable; translational neurophysiological studies may need prior strokes to be fully excluded

5. Multisite trials selecting sites for trials need to evaluate screening data from local studies to determine site-specific barriers to recruitment, transfer from acute care to inpatient rehabilitation or community services which may enhance recruitment potential of a site.

\section{In Acute Care: Short Length of Stay, $m R S>1$, and Prior Stroke Limit Recruitment}

CPASS screening data from the acute care setting showed patients' length of stay varied from 6 days for the mildly impaired to 17 days for the severely impaired patients. On average, the moderately impaired were admitted for 13.6 days. A majority of these moderately-impaired (49\%) were discharged to Inpatient rehabilitation, which opened another window to recruit them for stroke trials, but the remaining $32 \%$ discharged to home (15\%) or to a skilled nursing facility (17\%) would be lost to recruitment forever unless a trial developed extensive outreach resources to recruit them from the community. These lengths of stay and discharge data are specific to an urban safety net hospital in the US where CPASS screening was conducted. Similar exhaustive data on recruitment for rehabilitation trials from across the US are missing at this time. Recently [29)], screening for acute stroke patients at the University Hospital Zurich in Switzerland reported on the eligibility criteria for a comparable UE trial. The typical length of stay (mean $=8$; range $4-12$ days; not stratified by severity as in the present report) was consistent with the brief length of stay we found with CPASS screening. It is critical for early stroke rehabilitation trials to develop efficient screening methods to rapidly identify eligible patients at the earliest time point after stroke. Other exclusion criteria that led to most exclusions in acute care included "rapidly improving motor function," "unable to follow two-step commands," "prior stroke," and "modified Rankin score (mRS)>1;" many of these are unmodifiable. Prior stroke led to exclusion of a large proportion of potentially eligible patients in our cohort. Similar findings have been reported in the European setting, 17\% excluded due to "recurrent stroke" (45). Given the incidence of recurrent and silent strokes, a judicious definition of "prior stroke" is necessary to not miss out on an otherwise large majority of eligible participants. For motor function studies, "prior strokes without residual impairment" may be an acceptable definition, although these depend on the specific research questions and trial phase. Similarly, a blanket exclusion based on mRS may be impractical for stroke rehabilitation trials since nearly $20 \%$ of all moderately impaired individuals were disqualified due to this single exclusion criteria.

\section{In Inpatient Rehabilitation: Too Mild, Prior Stroke, Arriving to Rehabilitation Too Late for The Study}

A large fraction of patients screened at inpatient rehabilitation were excluded because they were already out of the enrollment window when they arrived at the inpatient facility. CPASS enrolled patients <30-days post stroke, nearly $12 \%$ were disqualified because they could not be identified as eligible within this timeframe. Thus, it is recommended to screen eligible patients while they are still admitted to acute care; it is crucial to develop streamlined screening, chart review, and identification of potentially eligible patients at the earliest. Inability to consent due to language or aphasia led to another potentially modifiable exclusion criteria ( $7 \%$ in mildly impaired; $9 \%$ in severely impaired).

Nearly $62 \%$ of individuals with ischemic stroke arriving to inpatient rehabilitation were "too mild" (NIHSS motor arm item $<1$ ) for an early neurorehabilitation trial and thus disqualified from CPASS. Given the large percentage disqualified due to this single exclusion criteria, we examined patients' arm impairment using a dedicated motor function impairment test, the ARAT. ARAT evaluates fine motor function and reaching to grasp and ARAT sub-scores are highly relevant to UE stroke trials. The mean ARAT score in this cohort was $39.41 \pm 18.6$ ( $\max$ $=57$ ). The minimal clinically important difference (MCID), the minimal change in score for patients to perceive an improvement in their UE motor function with the ARAT is 5.7 points (46). Thus, although classified ineligible, individuals with an NIHSS motor arm score $<1$ show potential for UE motor recovery and are not at the ceiling of their UE motor function. Trials may benefit from performing additional screening using a motor function-specific measure like the ARAT or SAFE for a subset of individuals. This is particularly important because prior studies have also highlighted that disqualifying "too mild" impairment in stroke trials excludes large proportions of stroke patients $(41,45)$. Alternately, motor-specific (pre-screening) measures such as the SAFE score have shown good predictive validity at 1 year and may be more sensitive than the NIHSS motor item score or mRS in the context of UE neurorehabilitation trials $(47,48)$. Similar screening data (as presented in the present report) from trials implementing the SAFE score as a prescreening measure for trial recruitment will fill an important gap for trialists. Our data on NIHSS and ARAT highlight the need for a simple, quick, motor-function specific prescreening tool that is sensitive throughout the range of UE motor impairment and shows 
good predictive validity for motor outcomes at 12-months or more post stroke (the typical primary outcome in post-stroke UE trials).

\section{LIMITATIONS}

Although we present a first set of exhaustive data on trial recruitment statistics in an early subacute stroke rehabilitation trials in the US, it must be noted that these recruitment data come from a single-site phase II stroke trial. Patients were screened at a single site, an urban safety net hospital and inpatient rehabilitation facility and as such is limited to trial recruitment and patient behaviors within the US. Generalizability to other recruitment settings needs more data. Patients were screened using the motor arm item of the NIHSS, which was adequate for CPASS inclusion/exclusion criteria as shown by CPASS recruitment rates that remained comparable to similar trials, but trialists should consider adding a more sensitive motor-specific screening tool such as the SAFE score or ARAT given the relatively large numbers of patients' with demonstrated impairment on the ARAT excluded from the trial for being "too mild."

\section{CONCLUSIONS}

A large percentage of trial eligible patients are excluded because trialists do not get to patients in time, before discharge from acute care, and patients arrive to inpatients rehabilitation too late for trial recruitment. Trialists performing early stroke rehabilitation trials therefore need to develop streamlined screening and recruitment pipeline to engage patients while they are still in acute care. For a subset of mildly impaired

\section{REFERENCES}

1. Cramer SC. The EXCITE trial: a major step forward for restorative therapies in stroke. Stroke. (2007) 38:2204-5. doi: 10.1161/STROKEAHA.107.486555

2. Dromerick AW, Lang CE, Birkenmeier RL, Wagner JM, Miller JP, Videen TO, et al. Very Early Constraint-Induced Movement during Stroke Rehabilitation (VECTORS): A single-center RCT. Neurology. (2009) 73:195201. doi: 10.1212/WNL.0b013e3181ab2b27

3. Alcobendas-Maestro M, Esclarin-Ruz A, Casado-Lopez RM, MunozGonzalez A, Perez-Mateos G, Gonzalez-Valdizan E, et al. Lokomat roboticassisted versus overground training within 3 to 6 months of incomplete spinal cord lesion: randomized controlled trial. Neurorehabil Neural Rep. (2012) 26:1058-63. doi: 10.1177/1545968312448232

4. Nadeau SE, Wu SS, Dobkin BH, Azen SP, Rose DK, Tilson JK, et al. Effects of task-specific and impairment-based training compared with usual care on functional walking ability after inpatient stroke rehabilitation: LEAPS trial. Neurorehabil Neural Repair. (2013) 27:370-80. doi: 10.1177/1545968313481284

5. Winstein CJ, Wolf SL, Dromerick AW, Lane CJ, Nelsen MA, Lewthwaite $\mathrm{R}$, et al. Effect of a task-oriented rehabilitation program on upper extremity recovery following motor stroke: the ICARE randomized clinical trial. JAMA. (2016) 315:571-81. doi: 10.1001/jama.2016.0276

6. Jørgensen HS, Nakayama H, Raaschou HO, Vive-Larsen J, Støier M, Olsen TS. Outcome and time course of recovery in stroke Part I: outcome the copenhagen stroke study. Arch Phys Med Rehabil. (1995) 76:399-405. doi: 10.1016/S0003-9993(95)80567-2 patients, trialists will benefit from performing a motor function specific test like the ARAT in addition to using the NIHSS motor arm item during in-person screening for a subset of patients.

\section{DATA AVAILABILITY STATEMENT}

The raw data supporting the conclusions of this article will be made available by the authors, without undue reservation.

\section{ETHICS STATEMENT}

The studies involving human participants were reviewed and approved by the Institutional Review Board at the MedStar Health Research Institute. The trial was registered at http://www.clinicaltrials.gov (Identifier: NCT02235974). The patients/participants provided their written informed consent to participate in this study.

\section{AUTHOR CONTRIBUTIONS}

SG, PF, DE, and AD conceived the research. SG conducted the research. SG, $\mathrm{AD}$, and $\mathrm{DE}$ interpreted the findings. SG, PF, $\mathrm{DE}$, and $\mathrm{AD}$ wrote and approved the manuscript. All authors contributed to the article and approved the submitted version.

\section{FUNDING}

This work was supported by NINDS StrokeNet SCANR grant 1U10NS086513-01, American Heart Association 17POST33410485, NICHD/NCMRR K12HD093427, and the Greenberg Family Foundation.
7. Dromerick AW, Edwardson MA, Edwards DF, Giannetti ML, Barth J, Brady KP, et al. Critical Periods After Stroke Study: A phase II trial. Netherlands: Congress on Neurorehabilitation and Neural Repair, Maastrecht (2015).

8. Krakauer JW, Carmichael ST, Corbett D, Wittenberg GF. Getting neurorehabilitation right: what can be learned from animal models? Neurorehabil Neural Repair. (2012) 26:923-31. doi: 10.1177/1545968312440745

9. Biernaskie J, Chernenko G, Corbett D. Efficacy of rehabilitative experience declines with time after focal ischemic brain injury. J Neurosci. (2004) 24:1245-54. doi: 10.1523/JNEUROSCI.3834-03.2004

10. Bernhardt J, Hayward KS, Kwakkel G, Ward NS, Wolf SL, Borschmann K, et al. Agreed definitions and a shared vision for new standards in stroke recovery research: the stroke recovery and rehabilitation roundtable taskforce. Neurorehabil Neural Repair. (2017) 31:793-9. doi: 10.1177/1545968317732668

11. Bernhardt J, Borschmann K, Boyd L, Carmichael ST, Corbett D, Cramer SC, et al. Moving rehabilitation research forward: developing consensus statements for rehabilitation and recovery research. Neurorehabil Neural Repair. (2017) 31:694-8. doi: 10.1177/1545968317724290

12. Briggs DE, Felberg RA, Malkoff MD, Bratina P, Grotta JC. Should mild or moderate stroke patients be admitted to an intensive care unit? Stroke. (2001) 32:871-6. doi: 10.1161/01.STR.32.4.871

13. Wolf SL, Winstein CJ, Miller JP, Thompson PA, Taub E, Uswatte G, et al. Retention of upper limb function in stroke survivors who have received constraint-induced movement therapy: the EXCITE randomised trial. Lancet Neurol. (2008) 7:33-40. doi: 10.1016/S1474-4422(07) 70294-6 
14. Wolf SL, Thompson PA, Winstein CJ, Miller JP, Blanton SR, NicholsLarsen DS, et al. The EXCITE stroke trial: comparing early and delayed constraint-induced movement therapy. Stroke. (2010) 41:2309-15. doi: 10.1161/STROKEAHA.110.588723

15. Lang KC, Thompson PA, Wolf SL. The EXCITE trial: reacquiring upper-extremity task performance with early versus late delivery of constraint therapy. Neurorehabil Neural Repair. (2013) 27:654-63. doi: $10.1177 / 1545968313481281$

16. Lasagna L. Problems in publication of clinical trial methodology. Clin Pharmacol Ther. (1979) 25 (5 Pt 2):751-3. doi: 10.1002/cpt1979255part2751

17. Kasenda B, Von Elm E, You J, Blümle A, Tomonaga Y, Saccilotto R, et al. Prevalence, characteristics, and publication of discontinued randomized trials. JAMA. (2014) 311:1045-52. doi: 10.1001/jama.2014.1361

18. Edwardson MA, Zhong X, Fiandaca MS, Federoff HJ, Cheema AK, Dromerick AW. Plasma microRNA markers of upper limb recovery following human stroke. Sci Rep. (2018) 8:12558. doi: 10.1038/s41598-018-31020-5

19. Cramer SC, Wolf SL, Adams Jr HP, Chen D, Dromerick AW, Dunning $\mathrm{K}$, et al. Stroke recovery and rehabilitation research: issues, opportunities, and the national institutes of health stroke net. Stroke. (2017) 48:813-9. doi: 10.1161/STROKEAHA.116.015501

20. Brott T, Adams Jr HP, Olinger CP, Marler JR, Barsan WG, Biller J, et al. Measurements of acute cerebral infarction: a clinical examination scale. Stroke. (1989) 20:864-70. doi: 10.1161/01.STR.20.7.864

21. Lang CE, Wagner JM, Dromerick AW, Edwards DF. Measurement of upperextremity function early after stroke: properties of the action research arm test. Arch Phys Med Rehabil. (2006) 87:1605-10. doi: 10.1016/j.apmr.2006.09.003

22. McDonnell M. Action research arm test. Aust J Physiother. (2008) 54:220. doi: 10.1016/S0004-9514(08)70034-5

23. Nordin A, Alt Murphy M, Danielsson A. Intra-rater and inter-rater reliability at the item level of the action research arm test for patients with stroke. $J$ Rehabil Med. (2014) 46:738-45. doi: 10.2340/16501977-1831

24. Yozbatiran N, Der-Yeghiaian L, Cramer SC. A standardized approach to performing the action research arm test. Neurorehabil Neural Repair. (2008) 22:78-90. doi: 10.1177/1545968307305353

25. Dromerick AW, Edwards DF, Hahn M. Does the application of constraint-induced movement therapy during acute rehabilitation reduce arm impairment after ischemic stroke? Stroke. (2000) 31:2984-8. doi: 10.1161/01.STR.31.12.2984

26. Wolf SL, Winstein CJ, Miller JP, Taub E, Uswatte G, Morris D, et al. Effect of constraint-induced movement therapy on upper extremity function 3 to 9 months after stroke: the EXCITE randomized clinical trial. JAMA. (2006) 296:2095-104. doi: 10.1001/jama.296.17.2095

27. Bernhardt J, Langhorne P, Lindley RI, Thrift AG, Ellery F, Collier J, et al. Efficacy and safety of very early mobilisation within $24 \mathrm{~h}$ of stroke onset (AVERT): a randomised controlled trial. Lancet. (2015) 386:46-55. doi: 10.1016/S0140-6736(15)60690-0

28. Lo AC, Guarino PD, Richards LG, Haselkorn JK, Wittenberg GF, Federman DG, et al. Robot-assisted therapy for long-term upper-limb impairment after stroke. N Eng J Med. (2010) 362:1772-83. doi: 10.1056/NEJMoa0911341

29. Yelnik AP, Quintaine V, Andriantsifanetra C, Wannepain M, Reiner $\mathrm{P}$, Marnef $\mathrm{H}$, et al. AMOBES (active mobility very early after stroke): a randomized controlled trial. Stroke. (2017) 48:400-5. doi: 10.1161/STROKEAHA.116.014803

30. Kwakkel G, Winters C, van Wegen EE, Nijland RH, van Kuijk AA, Visser-Meily A, et al. Effects of unilateral upper limb training in two distinct prognostic groups early after stroke: the EXPLICIT-stroke randomized clinical trial. Neurorehabil Neural Repair. (2016) 30:804-16. doi: $10.1177 / 1545968315624784$

31. Cramer SC, Dodakian L, Le V, See J, Augsburger R, McKenzie A, et al. Efficacy of home-based telerehabilitation vs in-clinic therapy for adults after stroke: a randomized clinical trial. JAMA Neurol. (2019) 76:1079-87. doi: 10.1001/jamaneurol.2019.1604

32. Rodgers $\mathrm{H}$, Bosomworth $\mathrm{H}$, Krebs HI, van Wijck F, Howel D, Wilson N, et al. Robot assisted training for the upper limb after stroke (RATULS): a multicentre randomised controlled trial. Lancet. (2019) 394:51-62. doi: 10.1016/S0140-6736(19)31055-4

33. Saposnik G, Cohen LG, Mamdani M, Pooyania S, Ploughman M, Cheung D, et al. Efficacy and safety of non-immersive virtual reality exercising in stroke rehabilitation (EVREST): a randomised, multicentre, single-blind, controlled trial. Lancet Neurol. (2016) 15:1019-27. doi: 10.1016/S1474-4422(16) 30121-1

34. Pomeroy VM, Hunter SM, Johansen-Berg H, Ward NS, Kennedy N, Chandler E, et al. Functional strength training versus movement performance therapy for upper limb motor recovery early after stroke: a RCT. Effic Mechan Eval. (2018) 5:1-112. doi: 10.3310/eme05030

35. Brunner I, Skouen JS, Hofstad H, Aßmus J, Becker F, Sanders $\mathrm{AM}$, et al. Virtual reality training for upper extremity in subacute stroke (VIRTUES): a multicenter RCT. Neurology. (2017) 89:2413-21. doi: 10.1212/WNL.0000000000004744

36. Adie K, Schofield C, Berrow M, Wingham J, Humfryes J, Pritchard $\mathrm{C}$, et al. Does the use of Nintendo Wii Sports(TM) improve arm function? Trial of Wii(TM) in stroke: a randomized controlled trial and economics analysis. Clin Rehabil. (2017) 31:173-85. doi: 10.1177/02692155166 37893

37. Virani SS, Alonso A, Benjamin EJ, Bittencourt MS, Callaway CW, Carson $\mathrm{AP}$, et al. Heart disease and stroke statistics-2020 update: a report from the American heart association. Circulation. (2020) 141:e139-596. doi: 10.1161/CIR.0000000000000757

38. Kwakkel G, Kollen BJ, van der Grond J, Prevo AJ. Probability of regaining dexterity in the flaccid upper limb: impact of severity of paresis and time since onset in acute stroke. Stroke. (2003) 34:2181-6. doi: 10.1161/01.STR.0000087172.16305.CD

39. Jørgensen HS, Nakayama H, Raaschou HO, Vive-Larsen J, Støier M, Olsen TS. Outcome and time course of recovery in stroke Part II: time course of recovery the copenhagen stroke study. Arch Phys Med Rehabil. (1995) 76:406-12. doi: 10.1016/S0003-9993(95)80568-0

40. Jørgensen HS, Nakayama H, Raaschou HO, Olsen TS. Stroke: neurologic and functional recovery the copenhagen stroke study. Physical Med Rehab Clin. (1999) 10:887-906. doi: 10.1016/S1047-9651(18)30169-4

41. Feldman WB, Kim AS, Chiong W. Trends in recruitment rates for acute stroke trials 1990-2014. Stroke. (2017) 48:799-801. doi: 10.1161/STROKEAHA.116.014458

42. Kwah LK, Herbert RD. Prediction of walking and arm recovery after stroke: a critical review. Brain Sci. (2016) 6:53. doi: 10.3390/brainsci6040053

43. Hawe RL, Scott SH, Dukelow SP. Taking proportional out of stroke recovery. Stroke. (2018) 50:204-11. doi: 10.1161/STROKEAHA.118.023006

44. Cumming TB, Thrift AG, Collier JM, Churilov L, Dewey HM, Donnan GA, et al. Very early mobilization after stroke fast-tracks return to walking: further results from the phase II AVERT randomized controlled trial. Stroke. (2011) 42:153-8. doi: 10.1161/STROKEAHA.110.594598

45. Held JP, van Duinen J, Luft AR, Veerbeek JM. Eligibility screening for an early upper limb stroke rehabilitation study. Front Neurol. (2019) 10:683. doi: $10.3389 /$ fneur.2019.00683

46. Lang CE, Edwards DF, Birkenmeier RL, Dromerick AW. Estimating minimal clinically important differences of upper-extremity measures early after stroke. Arch Phys Med Rehabil. (2008) 89:1693-700. doi: 10.1016/j.apmr.2008.02.022

47. Stinear CM, Byblow WD, Ackerley SJ, Barber PA, Smith MC. Predicting recovery potential for individual stroke patients increases rehabilitation efficiency. Stroke. (2017) 48:1011-9. doi: 10.1161/STROKEAHA.116. 015790

48. Lang CE, DeJong SL, Beebe JA. Recovery of thumb and finger extension and its relation to grasp performance after stroke. J Neurophysiol. (2009) 102:451-9. doi: 10.1152/jn.91310.2008

Conflict of Interest: The authors declare that the research was conducted in the absence of any commercial or financial relationships that could be construed as a potential conflict of interest.

Copyright $\odot 2021$ Geed, Feit, Edwards and Dromerick. This is an open-access article distributed under the terms of the Creative Commons Attribution License (CC BY). The use, distribution or reproduction in other forums is permitted, provided the original author(s) and the copyright owner(s) are credited and that the original publication in this journal is cited, in accordance with accepted academic practice. No use, distribution or reproduction is permitted which does not comply with these terms. 